\title{
Aging Affects Neural Precision of Speech Encoding
}

\author{
Samira Anderson, ${ }^{1,2}$ Alexandra Parbery-Clark, ${ }^{1,2}$ Travis White-Schwoch, ${ }^{1,2}$ and Nina Kraus ${ }^{1,2,3,4,5}$ \\ ${ }^{1}$ Auditory Neuroscience Laboratory, Departments of ${ }^{2}$ Communication Sciences, ${ }^{3}$ Neurobiology and Physiology, and ${ }^{4}$ Otolaryngology, and \\ ${ }^{5}$ Interdepartmental Neuroscience Program, Northwestern University, Evanston, Illinois 60208
}

Older adults frequently report they can hear what is said but cannot understand the meaning, especially in noise. This difficulty may arise from the inability to process rapidly changing elements of speech. Aging is accompanied by a general slowing of neural processing and decreased neural inhibition, both of which likely interfere with temporal processing in auditory and other sensory domains. Age-related reductions in inhibitory neurotransmitter levels and delayed neural recovery can contribute to decreases in the temporal precision of the auditory system. Decreased precision may lead to neural timing delays, reductions in neural response magnitude, and a disadvantage in processing the rapid acoustic changes in speech. The auditory brainstem response (ABR), a scalp-recorded electrical potential, is known for its ability to capture precise neural synchrony within subcortical auditory nuclei; therefore, we hypothesized that a loss of temporal precision results in subcortical timing delays and decreases in response consistency and magnitude. To assess this hypothesis, we recorded ABRs to the speech syllable /da/ in normal hearing younger (18-30 years old) and older (60 - 67 years old) adult humans. $01 \mathrm{der}$ adults had delayed ABRs, especially in response to the rapidly changing formant transition, and greater response variability. We also found that older adults had decreased phase locking and smaller response magnitudes than younger adults. Together, our results support the theory that older adults have a loss of temporal precision in the subcortical encoding of sound, which may account, at least in part, for their difficulties with speech perception.

\section{Introduction}

As the aging population grows, the communication problems experienced in later stages of life are more prevalent. Older adults often report that they can hear what their communication partner is saying but that they cannot understand the meaning. In other words, the problem is not simply one of audibility but of clarity. Accurate speech discrimination requires precise temporal processing, an ability that is compromised in older adults compared with younger adults. Speaking at slower rates improves intelligibility for older adults (Craig, 1992), supporting the idea that overall age-related slowing contributes to problems with speech perception (Wingfield et al., 1999; Tremblay et al., 2002). Neurophysiological evidence of delayed neural timing and decreased temporal processing ability with age has been found in animals (Walton et al., 1998; Burkard and Sims, 2001; Finlayson, 2002; Recanzone et al., 2011) and humans (Burkard and Sims, 2001; Caspary et al., 2005; Lister et al., 2011; Vander Werff and Burns, 2011; Wang et al., 2011; Konrad-Martin et al., 2012; Parbery-Clark et al., 2012). For example, Burkard and Sims (2001) found prolonged latencies in the auditory brainstem re-

\footnotetext{
Received May 6, 2012; revised July 31, 2012; accepted Aug. 4, 2012.

Author contributions: S.A., A.P.-C., and N.K. designed research;S.A., A.P.-C., and T.W.-S. performed research; S.A. and T.W.-S. analyzed data; S.A., A.P.-C., T.W.-S., and N.K. wrote the paper.

This work is supported by National Institutes of Health Grants T32 DC009399 and R01 DC010016. We thank Sarah Drehobl and Emily Hittner for their assistance with data collection and analysis and members of the Auditory Neuroscience Laboratory for their feedback. We express our appreciation to Adam Tierney and Trent Nicol for developing the codes used for the phase locking and response consistency analyses, respectively. We also thank Erika Skoe, Jane Hornickel, and Trent Nicol for their comments on this manuscript.

Correspondence should be addressed to Dr. Nina Kraus, 2240 Campus Drive, Evanston, IL 60208. E-mail: nkraus@northwestern.edu.

DOI:10.1523/JNEUROSCI.2176-12.2012

Copyright $\odot 2012$ the authors $\quad 0270-6474 / 12 / 3214156-\bullet \$ 15.00 / 0$
}

sponse (ABR) to clicks in older relative to younger adults but only at fast stimulation rates, affirming the idea that temporal processing of rapid stimuli is compromised in older adults. What remains unknown is the effect of aging on subcortical processing of specific speech cues, such as timing, frequency, and harmonics, in older participants who are relatively free of hearing loss.

Older adults' neural responses may be affected by decreased levels of inhibitory neurotransmitters, a consistent finding in the dorsal cochlear nuclei (Caspary et al., 2005; Wang et al., 2009), inferior colliculi (ICs) (Caspary et al., 1995), and auditory cortices (de Villers-Sidani et al., 2010; Hughes et al., 2010; JuarezSalinas et al., 2010) of aging animals. Speech is temporally dynamic (Liederman et al., 2005); accurate processing of the rapidly changing aspects of speech depends in part on the sharpening of neural responses through inhibitory mechanisms (Walton et al., 1998; Caspary et al., 2002, 2008). The synchronous neural firing of the auditory brainstem results in a precise temporal representation of incoming sound (Kraus et al., 2000), but this precision may be degraded by decreased inhibition or other factors association with aging, such as variability in neural firing (Turner et al., 2005; Yang et al., 2009) or temporal jitter (PichoraFuller et al., 2007). Indeed, temporal jitter or lack of neural response consistency is thought to contribute to impairments in binaural processing (Pichora-Fuller and Schneider, 1992) and speech perception deficits in older adults (Pichora-Fuller et al., 2007).

We hypothesized that the consequences of reductions in inhibitory neurotransmitters in older adults include the following: (1) delayed neural timing (i.e., delayed peak latencies); (2) greater variability in neural firing, reflected in decreased intertrial response consistency; and (3) decreased phase locking and spectral 
Table 1. Participant characteristics

\begin{tabular}{llr}
\hline & Younger $(n=17)$ & Older $(n=17)$ \\
\hline $\begin{array}{l}\text { Age (years) } \\
\text { Hearing thresholds (dB HL) }\end{array}$ & $23.18(3.52)$ & $62.00(2.24)$ \\
$125 \mathrm{~Hz}$ & $7.94(4.61)$ & $10.00(3.42)$ \\
$250 \mathrm{~Hz}$ & $7.06(3.45)$ & $8.68(2.81)$ \\
$500 \mathrm{~Hz}$ & $7.79(3.63)$ & $8.68(2.95)$ \\
$1000 \mathrm{~Hz}$ & $7.21(2.48)$ & $7.06(3.45)$ \\
$2000 \mathrm{~Hz}$ & $6.62(3.42)$ & $8.09(3.91)$ \\
$4000 \mathrm{~Hz}$ & $4.56(3.22)$ & $12.06(3.67)$ \\
$8000 \mathrm{~Hz}$ & $6.18(3.55)$ & $18.53(7.13)$ \\
Click (ms) & $1.71(0.12)$ & $1.77(0.21)$ \\
I & $3.89(0.13)$ & $4.01(0.27)$ \\
III & $5.69(0.24)$ & $5.90(0.44)$ \\
V & $69.3(16.16)$ & $72.0(25.84)$ \\
IQ percentiles & & \\
\hline Means (with SDs) for the younger and older groups are listed for age, hearing thresholds (125-8000 Hz, dB HL), click \\
waves I,III, and Vlatencies, and non-verbal IQ percentiles (young adults, Test of Nonverbal Intelligence; older adults, \\
Wechsler Abbreviated Scale of Intelligence Matrix Reasoning Subtest). The Test of Nonverbal Intelligence and \\
Wechsler Abbreviated Scale of Intelligence scores were converted to percentiles to facilitate comparison.
\end{tabular}

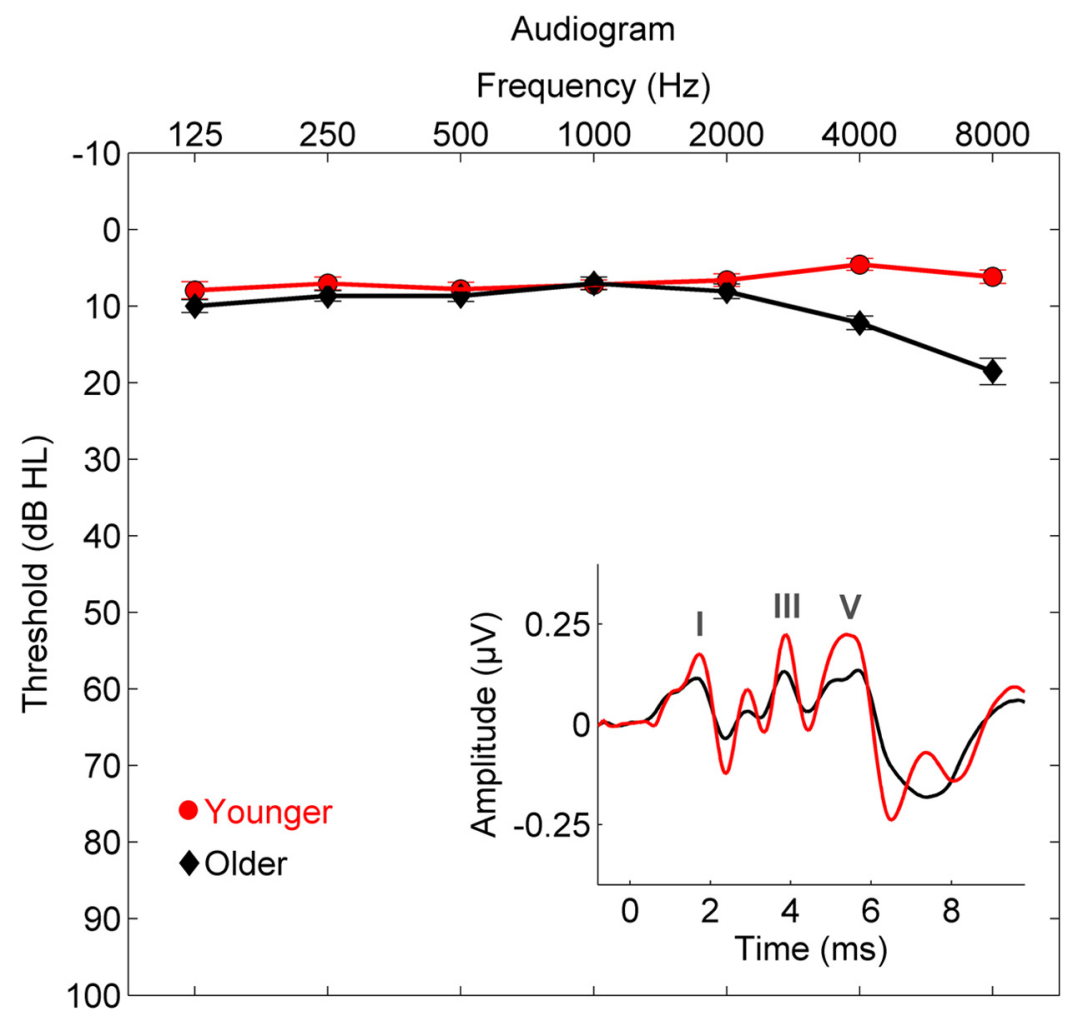

Figure 1. Average hearing thresholds are plotted from 125 to $8000 \mathrm{~Hz}$ for younger (red circles) and older (black diamonds) adults, with error bars indicating \pm 1 SE. Hearing thresholds between the two groups are equivalent, except at 4000 and $8000 \mathrm{~Hz}$ (both $p<0.001$ ). Inset, average click-evoked waveforms for younger and older adults. Latencies are equivalent for waves I and III; there is a trending difference for wave $V(p=0.083)$.

magnitudes. Here, we compared subcortical processing of a speech syllable in younger and older adults using the ABR to complex sounds (cABR), a method that has proven useful in older adults and other populations (Anderson et al., 2011; Vander Werff and Burns, 2011). Establishing the biological consequences of age-related neural slowing on subcortical speech encoding will help elucidate inferred mechanisms underlying impaired speech-in-noise (SIN) perception in older adults.

\section{Materials and Methods \\ Participants}

Participants comprised 17 young adults (18-30 years old; mean \pm SD, $23.18 \pm 3.52$ years; four males) and 17 older adults (60-67 years old; mean $\pm \mathrm{SD}, 62 \pm 2.24$; three males) recruited from the Chicago area. All participants had clinically normal hearing, defined as follows: (1) air conduction thresholds $\leq 20 \mathrm{~dB}$ HL from 125 to $4000 \mathrm{~Hz}$ bilaterally ( $\leq 25$ $\mathrm{dB} \mathrm{HL}$ at $8000 \mathrm{~Hz}$ ); (2) air-bone gaps $\leq 10 \mathrm{~dB} \mathrm{HL}$; and (3) no interaural asymmetry ( $\geq 15 \mathrm{~dB}$ HL difference at two or more frequencies). Additionally, all subjects had normal click-evoked brainstem response latencies (wave $\mathrm{V}<6.8 \mathrm{~ms}$ ), measured by a $100 \mu$ s click stimulus presented at $80 \mathrm{~dB}$ SPL at a rate of $31.4 \mathrm{~Hz}$. No participants reported a history of neurologic conditions. Because extensive musical training and bilingualism can affect auditory brainstem processing across the lifetime (Parbery-Clark et al., 2009; Bidelman and Krishnan, 2010; Krizman et al., 2012; Parbery-Clark et al., 2012; for review, see Kraus and Chandarsekaran, 2010), anyone who reported a history of musical training ( $>3$ years) or was a non-native English speaker was excluded.

Participants had normal IQ scores [young adults, $\geq 85$ on the Test of Nonverbal Intelligence (Brown et al., 1997); older adults, $\geq 85$ on the Wechsler Abbreviated Scale of Intelligence (Zhu and Garcia, 1999)]. Different IQ screening tests were used for these age groups because we combined datasets from two studies for the aging analysis. In addition, the older adults were screened for dementia using a cutoff score of 22/30 on the Montreal Cognitive Assessment (MOCA) (Nasreddine et al., 2005). Groups were sex matched (Fisher's exact test, $p=0.656)$ and matched audiometrically through $2000 \mathrm{~Hz}$ ( $t$ tests, all $p>0.1$ ). Although there were significant group differences for hearing thresholds at 4000 and $8000 \mathrm{~Hz}(p<$ 0.001 ), the difference at $4000 \mathrm{~Hz}$ was within the margin of error of the audiogram $(\sim 5 \mathrm{~dB} \mathrm{HL})$, and the difference at $8000 \mathrm{~Hz}$ was $\sim 12 \mathrm{~dB} \mathrm{HL}$. See Table 1 for participant characteristics in each group and Figure 1 for average audiometric thresholds and click latencies. All procedures were reviewed and approved by the Institutional Review Board of Northwestern University. Participants gave informed consent and were paid for their time.

\section{Electrophysiology}

Stimulus and recording. A $170 \mathrm{~ms}$ speech syllable, /da/, was synthesized at a $20 \mathrm{kHz}$ sampling rate with a Klatt-based synthesizer (Klatt, 1980). After an initial $5 \mathrm{~ms}$ stop burst in the syllable, voicing remained constant with a fundamental frequency $\left(\mathrm{F}_{0}\right)$ of $100 \mathrm{~Hz}$. During the $50 \mathrm{~ms}$ transition from the $/ \mathrm{d} /$ to the $/ \mathrm{a} /$, the lower three formants shifted $\left(\mathrm{F}_{1}, 400 \rightarrow 720\right.$ $\left.\mathrm{Hz} ; \mathrm{F}_{2}, 1700 \rightarrow 1240 \mathrm{~Hz} ; \mathrm{F}_{3}, 2580 \rightarrow 2500 \mathrm{~Hz}\right)$ but stabilized for the $120 \mathrm{~ms}$ steady-state vowel portion. The fourth through sixth formants $\left(\mathrm{F}_{4}-\mathrm{F}_{6}\right)$ remained constant over $170 \mathrm{~ms}$ at 3300, 3750, and $4900 \mathrm{~Hz}$, respectively. The /da/ was chosen because it combines a transient (the /d/) and periodic (the /a/) segment, two acoustic features that have been extensively studied in cABRs (Skoe and Kraus, 2010). Additionally, stop consonants pose challenges to young and old listeners (Miller and Nicely, 1955) and thus provide a more ecologically valid stimulus than other complex sounds, such as tones. Finally, the / da/ has been shown to elicit a robust and replicable cABR across the lifespan (Hornickel et al., 2009; Parbery-Clark et al., 2012). A waveform of the /da/ is presented in Figure $2 A$, along with average responses in younger and older adults $(C)$. A spectrogram is shown in Figure $2 B$. The /da/ was presented binaurally using Neuroscan $\operatorname{Stim}^{2}$ (Compumedics) with alternating polarities at 80 
dB SPL at a rate of $3.95 \mathrm{~Hz}$ through electromagnetically shielded insert earphones (ER-3; Etymotic Research). Subcortical responses were digitized at $20 \mathrm{kHz}$. Electromagnetically shielded insert earphones were used to reduce stimulus and noise artifact. A vertical montage of $4 \mathrm{Ag}-\mathrm{AgCl}$ electrodes $(\mathrm{Cz}$ active, forehead ground, earlobe references) was used with all impedances $<5 \mathrm{k} \Omega$. Continuous responses were recorded with Neuroscan Acquire 4.3. During the recording session $(\sim 28 \mathrm{~min})$, participants sat in a recliner and watched a silent, captioned film of their choice to facilitate a relaxed yet wakeful state. Six thousand artifactfree sweeps were recorded from each participant.

Data reduction. Responses were digitally bandpass filtered offline from 70 to $2000 \mathrm{~Hz}$ using Neuroscan Edit. This frequency range filters out cortical activity while maximizing signal-to-noise ratio (SNR) and the detection of transient peaks (such as the onset). A Butterworth filter with a $12 \mathrm{~dB} /$ octave rolloff and zero phase shift was applied, and then responses were epoched using a -40 to $213 \mathrm{~ms}$ time window referenced to the stimulus onset. Any sweep with amplitude greater than $\pm 35 \mu \mathrm{V}$ was considered artifact and rejected before averaging. Final averages comprised 6000 sweeps (3000 of each polarity). Two final average responses were created for subsequent analysis. In the first, the two polarities were added to minimize the influence of cochlear microphonic and stimulus artifact on the response (Gorga et al., 1985; Campbell et al., 2012) and to maximize the envelope response. In the second average, the two polarities were subtracted to enhance temporal fine structure in the harmonics (formant-related harmonics) (Aiken and Picton, 2008; Hornickel et al., 2012). Added responses were used to analyze latency, amplitude, frequency representation of the fundamental frequency and lower harmonics, phase-locking factor, and response consistency; subtracted polarities were used to analyze the frequency representation of the first formant $(400-720 \mathrm{~Hz})$. Before analysis, responses were amplitude baselined in the prestimulus period.

\section{Analyses}

Timing. To analyze the effects of age on neural timing, we manually identified peaks in the subcortical responses. The identification provides the latency and amplitude of each peak. Peaks were labeled according to a reference latency (i.e., a peak occurring 33-34 ms after onset would be called "peak 33"; see Fig. 3). The onset peak was identified as peak 9 , transition peaks were $33,43,53$, and 63 , and steady-state peaks were $73,83,93, \ldots, 163$. Note that we included the $63 \mathrm{~ms}$ peak in the transition analysis, because we observed that the periodicity in the older adults' responses did not become stable until $70 \mathrm{~ms}$. Two trained peak pickers, blind to participant group, identified each major peak of interest in the onset, transition, and steady-state portions of the response. An additional trained peak picker confirmed each peak identification. We also identified peaks for waves I, III, and V of click-evoked responses.

Response consistency. We computed a measure of response consistency over the length of the recording period. Filtered, epoched, and baselined responses were reaveraged offline in MATLAB (MathWorks). Two "paired" averages from an individual response were computed. Each

A
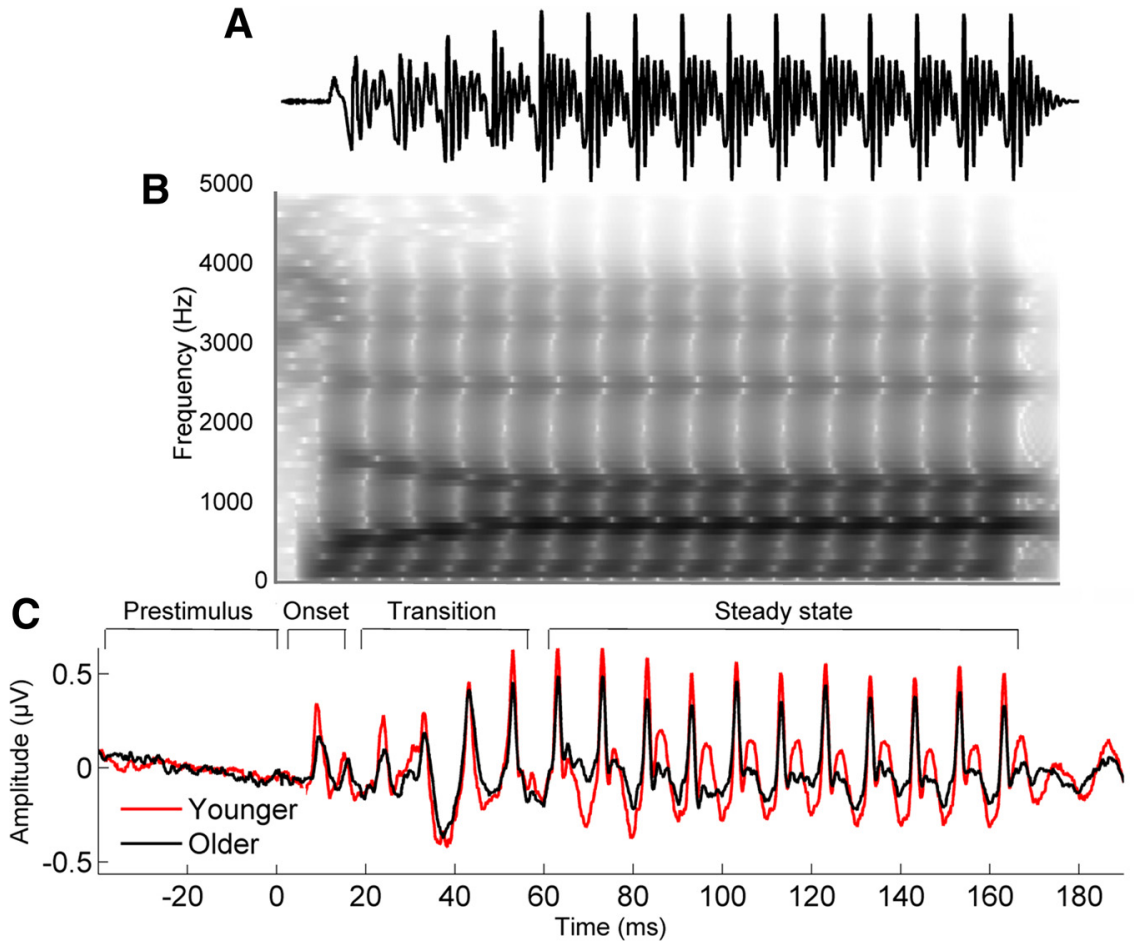

Figure 2. $\quad \boldsymbol{A}, \boldsymbol{B}$, Stimulus waveform $(\boldsymbol{A})$ and spectrogram $(\boldsymbol{B})$ for the speech syllable/da/. $\boldsymbol{C}$, Average brainstem responses to /da/ in the younger (red) and older (black) adults. The prestimulus and response regions are labeled with respect to the onset, formant transition, and steady-state vowel of the stimulus.

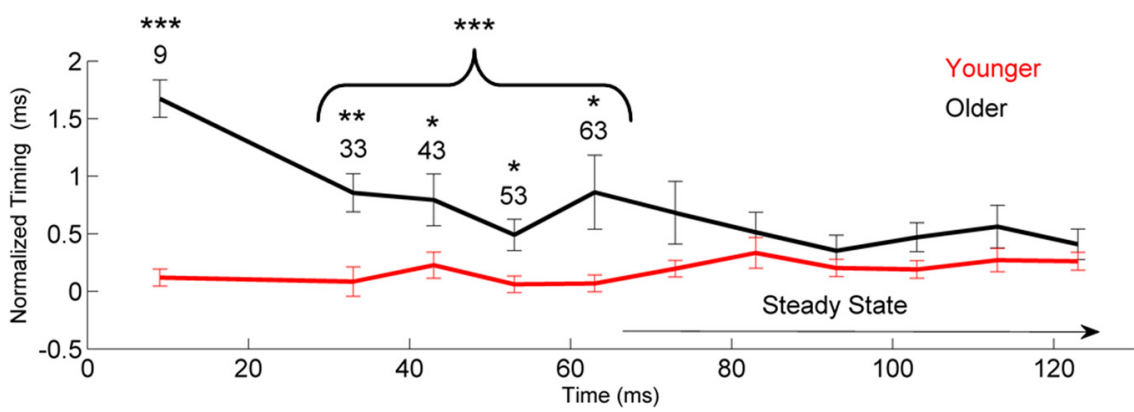

Figure 3. Neural delays in the aging population. Older adults show a shift in neural response timing for both the onset and transition but not for the steady state. To facilitate visualization of the data, the peak latencies are normalized. Normalization was

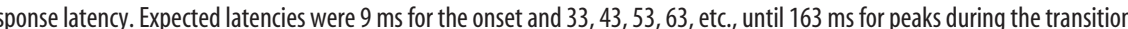
and steady state. This resulted in a normalized value (milliseconds), with 0 indicating that peak occurred at its expected latency. ${ }^{*} p<0.05,{ }^{* *} p<0.01,{ }^{* * *} p<0.001$. Error bars represent $\pm 1 \mathrm{SE}$.

average comprised 3000 randomly selected non-overlapping sweeps from the response (for example, if one average had sweeps $1,2,4,7, \ldots$, and 6000 , its companion average would have sweeps $3,5,6,8, \ldots$, and 5999). These two averages were correlated to compute a Pearson's correlation coefficient. This process was performed 300 times for each individual response, each time selecting random subsets of sweeps; the mean correlation of these 300 sets of paired averages was computed. Pearson's $r$ scores were Fisher transformed to $z^{\prime}$-scores for statistical purposes (Cohen and Cohen, 1975). Correlations were performed over three time ranges corresponding to the entire response (5-180 ms), the transition (20-60 ms), and the steady state (60-170 ms).

Phase-locking factor. To obtain a measure of phase-locking ability, we performed a wavelet analysis in MATLAB. This provides a measure of trial-to-trial phase coherence ("phase-locking factor") of encoding of the speech syllable within a specified time $\times$ frequency range of the response (Fell, 2007). The analysis procedure is similar to the wavelet analysis described by Tallon-Baudry et al. (1996). A complex Morlet (Gaussian- 
localized) wavelet function was convoluted with the response at each time point to provide an amplitude (arbitrary units) and phase (radians) value for each time $\times$ frequency bin in the response. The amplitude corresponds to the energy at that point in the signal and the phase to the direction of a momentary deflection in the EEG signal. This amplitude was normalized to 1 to weigh each trial equally, regardless of its magnitude (i.e., voltage). Resulting vectors were averaged to compute a complex value that represents phase distribution for each one of the 6000 trials of the /da/; the modulus (i.e., absolute magnitude) of this value is the resulting phase-locking factor. A phase-locking factor of 1 indicates perfect phase coherence at every point in every trial, whereas a phaselocking factor of 0 indicates no phase coherence at any point in every trial. In practice, the range of phase-locking factors is small (up to $\sim 0.15-$ 0.20 ) because of the relatively poor SNR offered by a single neural response trial in a far-field brainstem recording, resulting in the presence of multiple aberrant oscillatory deflections (cf. Ruggles et al., 2011, 2012). Phase-locking factors were calculated across the frequency range of $70-$ $800 \mathrm{~Hz}$ for the entire response (5-180 ms), the transition $(20-60 \mathrm{~ms})$, and the steady state $(60-170 \mathrm{~ms})$. Phase-locking factors were also computed for restricted frequency bins corresponding to the $\mathrm{F}_{0}(100 \mathrm{~Hz})$ and its integer harmonics $(200-700 \mathrm{~Hz})$ across the same three time ranges. Additionally, a three-dimensional plot was generated (see Fig. 5) with a color spectrum representing phase-locking power (warmer colors indicating stronger phase locking).

Response and spectral magnitudes. Root mean square (RMS) amplitude was used to objectively quantify the overall magnitude of response and prestimulus (i.e., nonresponse) activity. RMS amplitudes were computed for the prestimulus period $(-40$ to $0 \mathrm{~ms})$, the entire response (5-180 ms), the transition $(20-60 \mathrm{~ms})$, and the steady state $(60-170 \mathrm{~ms})$.

Average spectral amplitudes were calculated from each response; for each time region, zero padding was applied before a Fourier analysis to increase the resolution of the spectral display to $1 \mathrm{~Hz} /$ point. For statistical analyses, average amplitudes were computed over $20 \mathrm{~Hz}$ bins around the frequencies of interest, which included the fundamental frequency $\left(\mathrm{F}_{0}\right)$ and its integer harmonics up to $1000 \mathrm{~Hz}\left(\mathrm{H}_{2}-\mathrm{H}_{10}\right)$. This procedure was performed over the entire response, the transition, and the steady state for added and subtracted polarity averages.

Statistical analyses. All statistical analyses were conducted in SPSS version 18.0 (SPSS). Multivariate ANOVA was used for group comparisons (young vs old) of multiple peaks, multivariate analysis of covariance was used for RMS amplitudes, the $\mathrm{F}_{0}$, and its harmonics, and one-way ANOVAs were used for latencies of individual peaks, response consistency, and phase-locking factor. Levene's test was used to ensure homogeneity of variance for all measures, and Shapiro-Wilk tests were used to ensure that all variables were normally distributed. The assumption of homogeneity of variance was violated for the latency analysis; therefore, Pillai's trace criterion was used as a conservative model. Bonferroni corrections for multiple comparisons were applied as appropriate; $p$ values reflect two-tailed tests.

\section{Results}

\section{Timing}

As predicted, younger adults had earlier peak latencies but only for the region of the response corresponding to the onset of the syllable $\left(F_{(1,33)}=75.436, p<0.001\right)$ and rapidly changing formant transition (overall: $F_{(4,29)}=11.683, p<0.001$; peaks: 33 $\mathrm{ms}, F_{(1,33)}=13.597, p=0.001 ; 43 \mathrm{~ms}, F_{(1,33)}=5.039$, $p=0.038$; $53 \mathrm{~ms}, F_{(1,33)}=7.626, p=0.009 ; 63 \mathrm{~ms}, F_{(1,33)}=5.779, p=$ $0.022)$. In contrast, the response peaks corresponding to the unchanging steady-state region of the syllable (73-163 ms) were equivalent between groups (overall: $F_{(10,23)}=1.506, p=0.200$; no individual peak differed, all $p>0.1$ ). For a graphic representation of latency differences between groups, see Figure 3 and Table 2.

We also compared click-evoked ABRs to determine whether these latency differences were driven by peripheral changes in the auditory system. The groups were closely matched on wave I
Table 2. Mean latencies for peaks in the onset, transition, and steady-state regions of the brainstem response

\begin{tabular}{rrr}
\hline & \multicolumn{2}{l}{ Latency (ms) } \\
\cline { 2 - 3 } Peak & \multicolumn{1}{l}{ Younger } & \multicolumn{1}{c}{ Older } \\
\hline 9 & $9.12(0.31)$ & $10.67(0.67)$ \\
33 & $33.08(0.53)$ & $33.86(0.68)$ \\
43 & $43.32(0.47)$ & $43.79(0.93)$ \\
53 & $53.06(0.30)$ & $53.49(0.56)$ \\
63 & $63.07(0.30)$ & $63.86(1.33)$ \\
73 & $73.20(0.30)$ & $73.68(1.12)$ \\
83 & $83.48(1.12)$ & $83.66(1.21)$ \\
93 & $93.20(0.31)$ & $93.49(1.08)$ \\
103 & $103.44(1.08)$ & $103.61(1.05)$ \\
113 & $113.46(1.07)$ & $113.61(1.23)$ \\
123 & $123.48(1.09)$ & $123.56(1.11)$ \\
133 & $133.48(1.01)$ & $133.75(1.30)$ \\
143 & $143.50(1.09)$ & $143.66(1.15)$ \\
153 & $153.48(1.13)$ & $153.64(1.21)$ \\
163 & $163.49(1.21)$ & $163.68(1.34)$ \\
\hline
\end{tabular}

Each peak is identified by its number, and the average peak latencies and SDs are reported.

Table 3. Response consistency $r$ values and response magnitudes

\begin{tabular}{lllr}
\hline Time range & Younger & Older & $p$ value \\
\hline $\begin{array}{llll}\text { Consistency (Pearson's } r \text { ) } \\
\quad \text { Transition }(20-60 \mathrm{~ms})\end{array}$ & $0.91(0.04)$ & $0.80(0.23)$ & 0.001 \\
$\quad$ Steady state $(60-170 \mathrm{~ms})$ & $0.91(0.06)$ & $0.73(0.25)$ & $<0.001$ \\
$\quad$ Entire $(5-180 \mathrm{~ms})$ & $0.90(0.05)$ & $0.75(0.24)$ & $<0.001$ \\
RMS amplitude $(\mu \mathrm{V})$ & & & \\
$\quad$ Transition $(20-60 \mathrm{~ms})$ & $0.28(0.07)$ & $0.25(0.08)$ & 0.023 \\
$\quad$ Steady state $(60-170 \mathrm{~ms})$ & $0.28(0.07)$ & $0.22(0.09)$ & 0.005 \\
Entire $(5-180 \mathrm{~ms})$ & $0.27(0.07)$ & $0.22(0.08)$ & 0.007 \\
\hline
\end{tabular}

Response consistency scores (Pearson's $r$ values) and RMS amplitudes ( $\mu \mathrm{V}$ ): means (with SDs) for younger and older groups across multiple time ranges, with $p$ values corresponding to group differences for each time range.

$\left(t_{(33)}=1.069, p=0.293\right)$ and wave III $\left(t_{(33)}=1.603, p=0.119\right)$; however, consistent with the $/ \mathrm{da} /$ results, evidence of delayed neural timing was seen in a trending difference in wave $\mathrm{V}$ latencies $\left(t_{(33)}=1.792, p=0.083\right)$. To ensure that these results were not driven by high-frequency hearing differences, we reran the analysis covarying for thresholds at 4000 and $8000 \mathrm{~Hz}$ and found that the trending difference in wave $\mathrm{V}$ latencies was still present $\left(t_{(30)}=1.872, p=0.064\right)$. See Table 1 for mean click latencies and Figure 1 for a comparison of average click-evoked waveforms for each group.

\section{Response consistency}

There were striking differences in response consistency between younger and older adults, whereby older adults had substantially less consistent responses. This was the case for the entire response $\left(F_{(1,33)}=18.792, p<0.001\right)$, the transition $\left(F_{(1,33)}=12.167, p=\right.$ $0.001)$, and the vowel $\left(F_{(1,33)}=17.692, p<0.001\right.$. $)$ To ensure that this effect was not driven by differences in levels of resting neural noise, we reran the analyses by covarying for prestimulus RMS amplitude and found no differences in the results. Mean correlation values and SDs are reported in Table 3.

\section{Phase locking}

Younger adults had better neural phase locking to the stimulus. When the phase-locking factor was averaged across a broad frequency range $(70-800 \mathrm{~Hz})$, there were substantial differences in the transition $\left(F_{(1,33)}=16.252, p<0.001\right)$, the steady state $\left(F_{(1,33)}=16.287, p<0.001\right)$, and the entire response $\left(F_{(1,33)}=\right.$ $18.468, p<0.001$; Table 4$)$. Average phase-locking factor is displayed for each group in Figure 4. 
Table 4. Phase-locking factors

\begin{tabular}{|c|c|c|c|c|c|c|c|c|c|}
\hline & \multicolumn{3}{|c|}{ Entire response (5-180 ms) } & \multicolumn{3}{|c|}{ Transition (20-60 ms) } & \multicolumn{3}{|c|}{ Steady state (60-170 ms) } \\
\hline & Younger & Older & $p$ & Younger & Older & $p$ & Younger & Older & $p$ \\
\hline $\mathrm{F}_{0}(80-120 \mathrm{~Hz})$ & $0.099(0.041)$ & $0.065(0.032)$ & 0.012 & $0.110(0.030)$ & $0.079(0.037)$ & 0.010 & $0.104(0.056)$ & $0.065(0.038)$ & 0.024 \\
\hline $\mathrm{H}_{2}(180-220 \mathrm{~Hz})$ & $0.071(0.019)$ & $0.042(0.026)$ & 0.001 & $0.062(0.022)$ & $0.038(0.018)$ & 0.001 & $0.081(0.027)$ & $0.048(0.033)$ & 0.003 \\
\hline $\mathrm{H}_{3}(280-320 \mathrm{~Hz})$ & $0.065(0.026)$ & $0.034(0.014)$ & $<0.001$ & $0.049(0.027)$ & $0.030(0.015)$ & 0.015 & $0.081(0.031)$ & $0.040(0.022)$ & $<0.001$ \\
\hline $\mathrm{H}_{4}(380-420 \mathrm{~Hz})$ & $0.060(0.027)$ & $0.036(0.018)$ & 0.005 & $0.045(0.017)$ & $0.029(0.013)$ & 0.004 & $0.074(0.038)$ & $0.043(0.024)$ & 0.008 \\
\hline $\mathrm{H}_{5}(480-520 \mathrm{~Hz})$ & $0.049(0.024)$ & $0.029(0.013)$ & 0.007 & $0.040(0.014)$ & $0.026(0.011)$ & 0.004 & $0.058(0.034)$ & $0.034(0.018)$ & 0.014 \\
\hline $\mathrm{H}_{6}(580-620 \mathrm{~Hz})$ & $0.027(0.001)$ & $0.020(0.007)$ & 0.012 & $0.025(0.006)$ & $0.020(0.007)$ & 0.041 & $0.030(0.013)$ & $0.021(0.009)$ & 0.024 \\
\hline $\mathrm{H}_{7}(680-720 \mathrm{~Hz})$ & $0.019(0.004)$ & $0.016(0.004)$ & 0.074 & $0.019(0.004)$ & $0.016(0.005)$ & 0.095 & $0.020(0.006)$ & $0.017(0.005)$ & 0.131 \\
\hline Overall $(70-800 \mathrm{~Hz})$ & $0.041(0.011)$ & $0.027(0.009)$ & $<0.001$ & $0.038(0.010)$ & $0.026(0.008)$ & $<0.001$ & $0.046(0.014)$ & $0.029(0.011)$ & $<0.001$ \\
\hline
\end{tabular}

Mean phase-locking factor (with SDs; arbitrary units) for the entire response, transition, and steady state in each group, with $p$ values corresponding to the group differences. Phase-locking factors are shown for the $\mathrm{F}_{0}$, each integer harmonic up to $\mathrm{H}_{7}$, and averaged across the entire response.

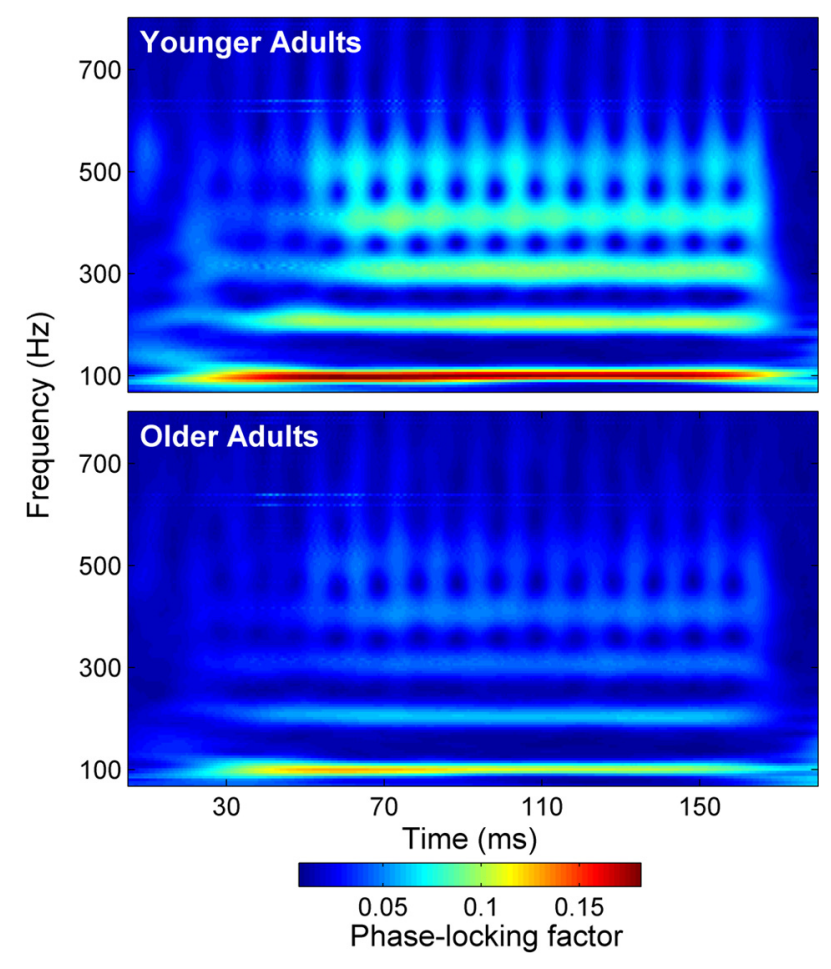

Figure 4. Phase locking factor. Top, younger adults. Bottom, older adults. Stronger phaselocking power is seen in younger adults, from the fundamental frequency through the upper harmonics and throughout the entire response.

\section{Response magnitude: time and frequency domains}

In the time domain, younger adults had greater RMS amplitudes for regions of the cABR corresponding to both the transition and the steady state of the stimulus compared with older adults (transition: $F_{(1,32)}=5.694, p=0.023$; steady state: $F_{(1,32)}=8.947, p=$ 0.005 ; entire: $\left.F_{(1,32)}=8.511, p=0.007\right)$, when covarying for prestimulus RMS amplitude. Although in older adults the amplitudes of response regions were smaller, the amplitude of the prestimulus region was larger in the older group $\left(t_{(33)}=3.295\right.$, $p=0.002$ ) (Fig. 5A). The more robust response magnitudes in young adults were also reflected in the frequency domain as greater representation of the fundamental frequency $\left(\mathrm{F}_{0}\right)$ and lower harmonics in the added responses calculated over the entire response (overall: $F_{(3,30)}=3.434, p=0.030 ; \mathrm{F}_{0}: F_{(1,33)}=$ 3.881, $p=0.058 ; \mathrm{H}_{2}: F_{(1,33)}=7.458, p=0.010 ; \mathrm{H}_{3}: F_{(1,33)}=$ $10.548, p=0.003)$. However, these effects were not as strong when the transition $\left(\mathrm{F}_{0}: F_{(1,33)}=2.818, p=0.103 ; \mathrm{H}_{2}: F_{(1,33)}=\right.$ 5.421, $\left.p=0.043 ; \mathrm{H}_{3}: F_{(1,33)}=4.434, p=0.043\right)$ and steady state $\left(\mathrm{F}_{0}: F_{(1,33)}=0.158, p=0.694 ; \mathrm{H}_{2}: F_{(1,33)}=5.235, p=0.029 ; \mathrm{H}_{3}\right.$ : $\left.F_{(1,33)}=2.025, p=0.164\right)$ were analyzed separately.
Spectral magnitudes were also calculated using subtracted polarities to obtain an estimate of fine structure representation that is less influenced by the speech envelope (Aiken and Picton, 2008; Skoe and Kraus, 2010; Hornickel et al., 2012). Spectral magnitudes corresponding to the first formant $(400-700 \mathrm{~Hz})$ of the stimulus were smaller in older versus younger adults when covarying for RMS amplitude in the prestimulus period (overall: $F_{(4,29)}=6.707, p=$ $0.001 ; \mathrm{H}_{4}: F_{(1,33)}=14.617, p=0.001 ; \mathrm{H}_{5}: F_{(1,33)}=3.359, p=0.076$; $\left.\mathrm{H}_{6}: F_{(1,33)}=16.994, p<0.001 ; \mathrm{H}_{7}: F_{(1,33)}=3.225, p=0.082\right)$. The comparison was also significant for the steady state (overall: $F_{(1,33)}=$ 5.737, $p=0.002 ; \mathrm{H}_{4}: F_{(4,29)}=10.372, p=0.003 ; \mathrm{H}_{5}: F_{(4,29)}=4.003$, $p=0.054 ; \mathrm{H}_{6}: F_{(1,33)}=15.889, p<0.001 ; \mathrm{H}_{7}: F_{(1,33)}=1.619, p=$ 0.212 ) but was not for the transition (overall: $F_{(4,29)}=1.148, p=$ $0.354 ; \mathrm{H}_{4}: F_{(1,33)}=1.690, p=0.203 ; \mathrm{H}_{5}: F_{(1,33)}=2.201, p=0.148$; $\left.\mathrm{H}_{6}: F_{(1,33)}=1.234, p=0.275 ; \mathrm{H}_{7}: F_{(1,33)}=3.760, p=0.061\right)$. Average added and subtracted spectral magnitudes are displayed in Figure 5 .

\section{Repeat analyses excluding individuals with below normal scores on the MOCA}

Five of 17 older participants had scores below 27 on the MOCA (scores: 24, 25, 25, 26, 26), which can indicate the possibility of mild cognitive impairment (Nasreddine et al., 2005). To ensure that the cABR differences were not driven by cognitive impairment, we repeated the analyses excluding these five subjects. All of the effects held as follows (entire response unless noted): (1) timing differences in onset and transition $\left(F_{(5,23)}=13.670, p<0.001\right)$; (2) response consistency $\left(F_{(1,31)}=31.11, p<0.001\right)$; (3) phase locking $\left(F_{(1,31)}=\right.$ 9.773, $p=0.004)$, RMS amplitude $\left(F_{(1,30)}=4.991, p=0.013\right)$, spectral added (low frequencies; $F_{(3,24)}=3.965, p=0.020$ ), and spectral subtracted (first formant; $F_{(4,23)}=3.021, p=0.039$ ). We conclude, therefore, that the observed age-related declines were not driven by individuals who may be experiencing mild cognitive impairment.

\section{Discussion}

Our results indicate delayed neural timing in the brainstem response to the onset and formant transition of a speech syllable in older adults compared with younger adults. This lack of neural precision was corroborated by decreased response consistency and phase-locking power. We found smaller RMS and spectral magnitudes in the responses of older adults. Our results replicate and extend previous findings of age-related latency and amplitude changes in response to a $40 \mathrm{~ms}$ stimulus encompassing only the onset and the transition (Vander Werff and Burns, 2011). The current results are also relatively free of the audiometric threshold confounds often found in comparisons of auditory performance in younger and older adults. 

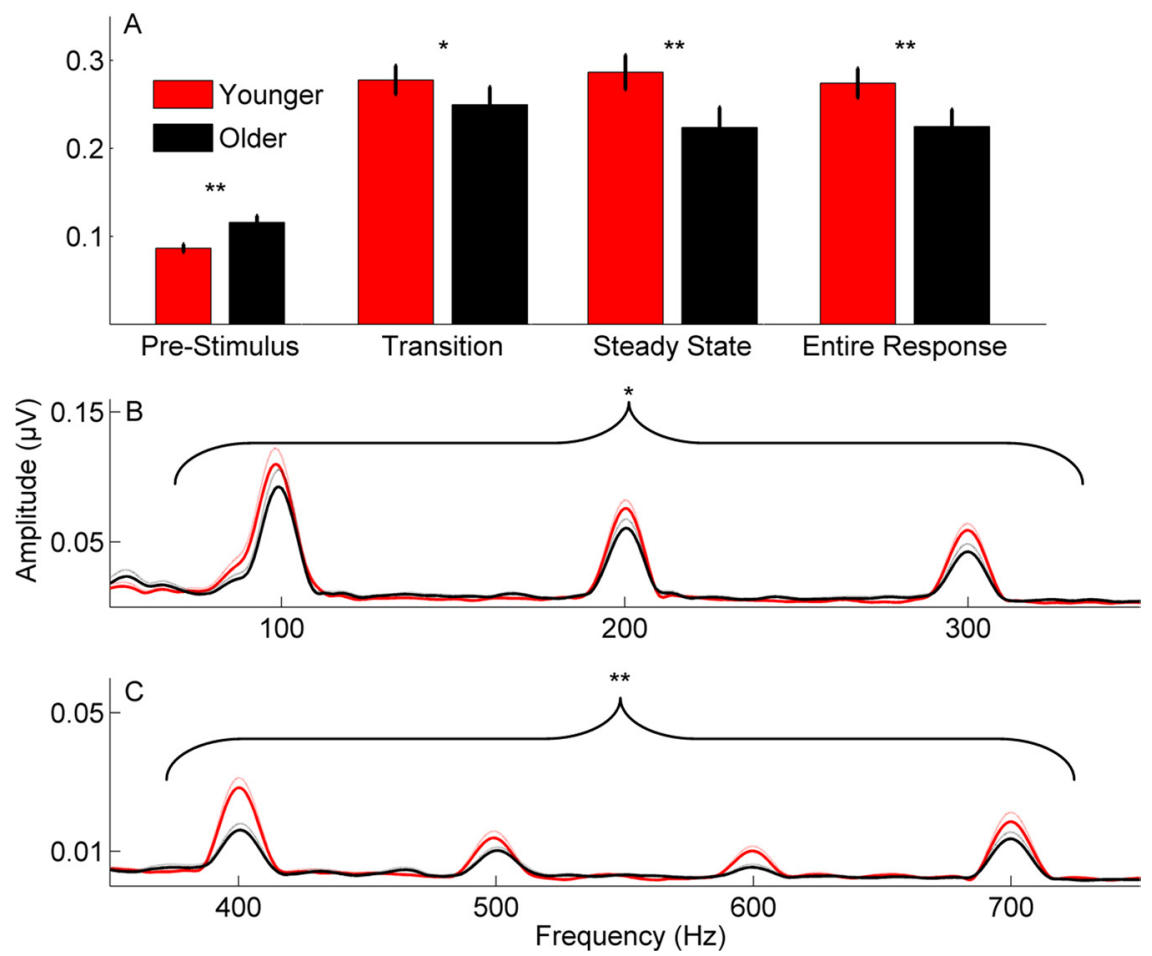

Figure 5. $\quad \boldsymbol{A}$, Bar graphs demonstrating significant between-group differences in RMS amplitude across stimulus time ranges The older adults have higher prestimulus activity as measured by RMS amplitude, yet the younger adults have higher RMS response amplitudes. $\boldsymbol{B}, \boldsymbol{C}$, Fast Fourier transforms calculated over the entire response. $\boldsymbol{B}$, Group average responses to added polarities in younger (red) versus older (black) adults. Greater magnitudes were noted in the $20 \mathrm{~Hz}$ bins surrounding lower frequencies, which contribute to pitch perception [fundamental frequency $\left(\mathrm{F}_{0}\right)$, second harmonic $\left(\mathrm{H}_{2}\right)$, and third harmonic $\left(\mathrm{H}_{3}\right)$ ]. $\mathrm{C}$, Group average responses to subtracted polarities in younger (red) versus older (black) adults. Greater magnitudes for younger adults were noted over the entire response but specifically for $\mathrm{H}_{4}-\mathrm{H}_{7}$ (the range encompassing the first formant). ${ }^{*} p<0.05,{ }^{* *} p<0.01$. Dotted lines and error bars represent $\pm 1 \mathrm{SE}$.

\section{Timing}

Using far-field electrophysiological techniques, we tested the inferred consequences of age-related decreases in inhibition on speech processing. Blocking inhibition in the IC reduces the selectivity required for discrimination of conspecific vocalizations in animal models (Klug et al., 2002), and GABAergic and glycinergic inhibition reduces the frequency selectivity of the IC (Koch and Grothe, 1998). The IC is the putative generator of the cABR (Chandrasekaran and Kraus, 2010); therefore, we propose that a reduction in inhibition alters subcortical temporal processing of the spectrotemporally dynamic formant transition.

The rapidly changing formants make the response to the transition vulnerable in noise, in contrast to the unchanging nature of the steady-state vowel (Anderson et al., 2010). Therefore, timing delays in the transition region likely lead to speech perception difficulties. The delays ranged from $1.5 \mathrm{~ms}$ in the onset and to $0.4-0.8 \mathrm{~ms}$ in transition. To put these results in context, interaural brainstem timing differences as small as $0.2 \mathrm{~ms}$ can be considered clinically significant when screening for cerebellopontine angle tumors (Grayeli et al., 2008), and differences on the order of 0.5-0.7 $\mathrm{ms}$ have separated good from poor perceivers of speech in noise (Anderson et al., 2010). Our finding of timing delays specific to the transition of the response indicate that aging selectively affects dynamic versus static encoding of speech processing. These selective timing differences in the transition may also arise from longer neural adaptation in older adults (Schneider and Hamstra, 1999). In the syllable /da/, the transition is shorter than the steady state (50 vs 120 $\mathrm{ms})$; therefore, the timing differences in the transition may arise from the older adult's longer adaptation period.
The lack of differences in click-evoked wave I and III latencies suggest that these deficits occur farther along the auditory pathway than the cochlea and auditory nerve. Evidence for age-related changes in brainstem structures is not new; amplitude reductions and lengthened wave I-V intervals in the click-evoked response were found in studies performed in the 1970s and 1980s (Fujikawa and Weber, 1977; Jerger and Hall, 1980; Maurizi et al., 1982).

\section{Response consistency and neural synchrony}

A decrease in the synchrony of neural firing may account for delayed neural timing and an overall decrease in representation of speech cues. We therefore assessed neural response consistency, expecting greater intertrial variability in responses in older adults. Indeed, older adults had less consistent brainstem responses than younger adults. Numerous studies of auditory perception have documented greater between-subject variability in older adults and have attributed this variability to differences in cognitive function (Harris et al., 2008; Rossi-Katz and Arehart, 2009; Arehart et al., 2011; Fogerty et al., 2010). Our analysis documents greater within-subject variability. Similar increases in response variability have been found in the primary visual cortex and middle temporal visual area in older monkeys (Yang et al., 2009) and the auditory cortex of older rats (Turner et al., 2005). These results support the idea raised by Pichora-Fuller et al. (2007), that temporal jitter in older adults contributes to difficulties with SIN perception. Additionally, response variability may have been a factor in studies showing decreased amplitudes (Parthasarathy et al., 2010) and a loss of temporal precision (Schatteman et al., 2008 ) in older versus younger animals. Indeed, Schatteman et al. (2008) conjectured that inhibitory neurotransmitters play a role in the synchronization of the brainstem to amplitude modulations, thus accounting for the loss of temporal resolution in older adults (Gordon-Salant and Fitzgibbons, 1993; Strouse et al., 1998; Schneider and Pichora-Fuller, 2001; Tremblay et al., 2002).

Variable neural firing in the auditory system leads to an imprecise representation of an auditory object, decreasing the listener's ability to selectively attend to a specific object from other auditory sources (Shinn-Cunningham and Best, 2008). When listening in noise, older adults draw on cognitive resources such as attention and memory more than younger adults (Wong et al., 2009), and cognitive demands increase reliance on perceptual cues (Kuo et al., 2011), activating efferent pathways (Nahum et al., 2008, Bajo et al., 2010; for review, see Kraus and Chandrasekaran, 2010). These findings, together with our results, suggest that older adults' perceptual impairments may arise in part from impaired subcortical representations that are modulated by cognitive influences. Perceptual problems may be exacerbated by age-related declines in higher-order cognitive functioning, re- 
ducing the compensatory gain afforded by cognitive influences (Davis et al., 2012).

The phase-locking analysis demonstrates deficits in the consistency of representation of stimulus phase. In the auditory cortex, phase tracking of speech envelopes is posited as a mechanism of speech intelligibility (Luo and Poeppel, 2007; Giraud and Poeppel, 2012). Our finding that older adults have lower phase locking suggests that this decline may be one mechanism contributing to poor SIN perception in older populations. Similar to the effects of decreased inhibition on encoding of rapidly changing temporal events, deterioration of phase locking may also rise from decreased inhibitory neurotransmitter levels (Koch and Grothe, 1998).

\section{Response magnitude: time and frequency domains}

In a previous study, we found that older adults with good SIN perception have larger RMS and $\mathrm{F}_{0}$ amplitudes than age- and hearing-matched adults with relatively poor SIN perception (Anderson et al., 2011); we conjectured that the older participants with poor SIN perception had experienced greater effects of biological aging, although they were matched for chronological age. Here, we directly tested that conjecture by comparing older and younger adults. We confirmed that aging affects RMS and $F_{0}$ amplitudes, reflecting decreased encoding of the speech envelope. Our results are consistent with previous findings indicating aging effects on phase coherence and amplitudes in brainstem responses to tone bursts (Clinard et al., 2010). In addition, the older adults' deficits in response to the stimulus fine structure provide a neural basis for the finding of age-related perceptual performance deficits in the use of fine structure cues (Vongpaisal and Pichora-Fuller, 2007; Grose and Mamo, 2010; Ben-David et al., 2011; Hopkins and Moore, 2011; Russo et al., 2012).

We found greater neural noise levels in our older adults during the prestimulus period. Similar results were found for click ABRs (Spivak and Malinoff, 1990): older adults had greater lowfrequency background noise in their responses. This increased noise may be attributable to a number of factors, including exogenous factors, such as greater muscle tension (Brown et al., 1999), and endogenous factors, such as increased spontaneous activity in the IC (Willott et al., 1988). Greater spontaneous neural activity has also been found in the auditory cortices of older rats (Hughes et al., 2010), especially layers I-III, in which higher numbers of inhibitory neurons are found (Prieto et al., 1994). Increased neural noise will decrease the SNR, providing at least a partial explanation for the older adult's speech perception difficulties.

\section{Clinical applications}

The older adults in our study all had normal hearing (thresholds $\leq 20 \mathrm{~dB}$ HL through $4000 \mathrm{~Hz}$ and $\leq 25 \mathrm{~dB} \mathrm{HL}$ at $8000 \mathrm{~Hz}$ ), potentially indicating that they are "biologically younger" than the majority of older people who experience some degree of peripheral presbycusis (Yueh et al., 2003). Despite this finding, our group of normal-hearing older adults still exhibited striking deficits in temporal processing; therefore, management of hearing difficulties in older adults must involve strategies extending beyond enhancing audibility. The benefits of training for improving the synchrony of neural firing have been demonstrated in rats (de Villers-Sidani et al., 2010), who achieved a partial reversal of behavioral deficits after being trained on a frequency discrimination task. Our results suggest that similar strategies should be considered when developing management plans for older adults who report difficulty hearing in noise. Studies with older musi- cians reinforce the idea that sustained, repeated cognitive engagement, such as is achieved through intensive musical training, offsets age-related subcortical timing delays (Parbery-Clark et al., 2012) and benefits SIN perception and temporal processing (Parbery-Clark et al., 2011; Zendel and Alain, 2012). Therefore, auditory training that engages memory and attention while training auditory discrimination is likely to improve auditory perception.

\section{Limitations}

Although both groups had normal audiometric thresholds through $4000 \mathrm{~Hz}(\leq 20 \mathrm{~dB} \mathrm{HL})$, there were significant group differences in the high frequencies $(4000$ and $8000 \mathrm{~Hz}$ ), which may have influenced our findings. Given that average hearing at these frequencies is highly related to age ( $\rho=0.746, p<0.001)$, controlling for thresholds would factor out our primary independent variable (age) and so was not a feasible resolution. Therefore, although we cannot fully rule out between-group differences in high-frequency hearing, we argue that these effects are, at least in part, driven by age-related biological declines.

\section{Conclusion}

We found evidence of diminished neural precision in the cABRs of older adults who had normal hearing across the audiometric frequency range, affirming the theory that suprathreshold temporal processing deficits contribute to the perceptual difficulties experienced by older adults (Ruggles et al., 2011; Shamma, 2011). One set of findings-age-related decreases in response consistency and phase locking - support the hypothesis that variability in neural firing leads to temporal processing deficits in older adults. Decreased inhibitory neurotransmission may be a factor in reduced ability to process rapid changes in speech stimuli. The neural timing delays were seen in response to the formant transition only, suggesting a selective timing deficit rather than a pervasive delay. Imprecise representation of an auditory signal reduces the ability to selectively attend to that signal and extract meaning from it. Work is currently underway to determine whether auditory training can partially offset these age-related changes in neural precision.

\section{References}

Aiken SJ, Picton TW (2008) Envelope and spectral frequency-following responses to vowel sounds. Hear Res 245:35-47.

Anderson S, Skoe E, Chandrasekaran B, Kraus N (2010) Neural timing Is linked to speech perception in noise. J Neurosci 30:4922-4926.

Anderson S, Parbery-Clark A, Yi HG, Kraus N (2011) A neural basis of speech-in-noise perception in older adults. Ear Hear 32:750-757.

Arehart KH, Souza PE, Muralimanohar RK, Miller CW (2011) Effects of age on concurrent vowel perception in acoustic and simulated electroacoustic hearing. J Speech Lang Hear Res 54:190-210.

Bajo VM, Nodal FR, Moore DR, King AJ (2010) The descending corticocollicular pathway mediates learning-induced auditory plasticity. Nat Neurosci 13:253-260.

Ben-David BM, Chambers CG, Daneman M, Pichora-Fuller MK, Reingold EM, Schneider BA (2011) Effects of aging and noise on real-time spoken word recognition: evidence from eye movements. J Speech Lang Hear Res 54:243-262.

Bidelman GM, Krishnan A (2010) Effects of reverberation on brainstem representation of speech in musicians and non-musicians. Brain Res 1355:112-125.

Brown L, Sherbenou R, Johnsen S (1997) Test of Nonverbal Intelligence (TONI-3). Austin, TX: Pro-Ed.

Brown M, Fisher JS, Salsich G (1999) Stiffness and muscle function with age and reduced muscle use. J Orthop Res 17:409-414.

Burkard RF, Sims D (2001) The human auditory brainstem response to high click rates: aging effects. Am J Audiol 10:53-61. 
Campbell T, Kerlin JR, Bishop CW, Miller LM (2012) Methods to eliminate stimulus transduction artifact from insert earphones during electroencephalography. Ear Hear 33:144-150.

Caspary DM, Milbrandt JC, Helfert RH (1995) Central auditory aging: GABA changes in the inferior colliculus. Exp Gerontol 30:349-360.

Caspary DM, Palombi PS, Hughes LF (2002) GABAergic inputs shape responses to amplitude modulated stimuli in the inferior colliculus. Hear Res 168:163-173.

Caspary DM, Schatteman TA, Hughes LF (2005) Age-related changes in the inhibitory response properties of dorsal cochlear nucleus output neurons: role of inhibitory inputs. J Neurosci 25:10952-10959.

Caspary DM, Ling L, Turner JG, Hughes LF (2008) Inhibitory neurotransmission, plasticity and aging in the mammalian central auditory system. J Exp Biol 211:1781-1791.

Chandrasekaran B, Kraus N (2010) The scalp-recorded brainstem response to speech: neural origins and plasticity. Psychophysiology 47:236-246.

Clinard CG, Tremblay KL, Krishnan AR (2010) Aging alters the perception and physiological representation of frequency: evidence from human frequency-following response recordings. Hear Res 264:48-55.

Cohen J, Cohen P (1975) Applied multiple regression/correlation analysis for the behavioral sciences. Hillsdale, NJ; Erlbaum.

Craig CH (1992) Effects of aging on time-gated isolated word-recognition performance. J Speech Hear Res 35:234-238.

Davis SW, Kragel JE, Madden DJ, Cabeza R (2012) The architecture of cross-hemispheric communication in the aging brain: linking behavior to functional and structural connectivity. Cereb Cortex 22:232-242.

de Villers-Sidani E, Alzghoul L, Zhou X, Simpson KL, Lin RC, Merzenich MM (2010) Recovery of functional and structural age-related changes in the rat primary auditory cortex with operant training. Proc Natl Acad Sci U S A 107:13900-13905.

Fell J (2007) Cognitive neurophysiology: beyond averaging. Neuroimage 37:1069-1072.

Finlayson PG (2002) Paired-tone stimuli reveal reductions and alterations in temporal processing in inferior colliculus neurons of aged animals. J Assoc Res Otolaryngol 3:321-331.

Fogerty D, Humes LE, Kewley-Port D (2010) Auditory temporal-order processing of vowel sequences by young and elderly listeners. J Acoust Soc Am 127:2509-2520.

Fujikawa SM, Weber BA (1977) Effects of increased stimulus rate on brainstem electric response (BER) audiometry as a function of age. J Am Audiol Soc 3:147-150.

Giraud AL, Poeppel D (2012) Cortical oscillations and speech processing: emerging computational principles and operations. Nat Neurosci 15:511-517.

Gordon-Salant S, Fitzgibbons PJ (1993) Temporal factors and speech recognition performance in young and elderly listeners. J Speech Hear Res 36:1276-1285.

Gorga M, Abbas P, Worthington D (1985) Stimulus calibration in ABR measurements. In: The auditory brainstem response (Jacobsen J, ed), pp 49-62. San Diego: College Hill.

Grayeli AB, Refass A, Smail M, Elgarem H, Kalamarides M, Bouccara D, Sterkers O (2008) Diagnostic value of auditory brainstem responses in cerebellopontine angle tumours. Acta Otolaryngol 128:1096-1100.

Grose JH, Mamo SK (2010) Processing of temporal fine structure as a function of age. Ear Hear 31:755-760.

Harris KC, Mills JH, He NJ, Dubno JR (2008) Age-related differences in sensitivity to small changes in frequency assessed with cortical evoked potentials. Hear Res 243:47-56.

Hopkins K, Moore BC (2011) The effects of age and cochlear hearing loss on temporal fine structure sensitivity, frequency selectivity, and speech reception in noise. J Acoust Soc Am 130:334-349.

Hornickel J, Skoe E, Nicol T, Zecker S, Kraus N (2009) Subcortical differentiation of stop consonants relates to reading and speech-in-noise perception. Proc Natl Acad Sci U S A 106:13022-13027.

Hornickel J, Anderson S, Skoe E, Yi HG, Kraus N (2012) Subcortical representation of speech fine structure relates to reading ability. Neuroreport 23:6-9.

Hughes LF, Turner JG, Parrish JL, Caspary DM (2010) Processing of broadband stimuli across A1 layers in young and aged rats. Hear Res 264:79-85.

Jerger J, Hall J (1980) Effects of age and sex on auditory brainstem response. Arch Otolaryngol 106:387-391.

Juarez-Salinas DL, Engle JR, Navarro XO, Recanzone GH (2010) Hierarchi- cal and serial processing in the spatial auditory cortical pathway is degraded by natural aging. J Neurosci 30:14795-14804.

Klatt D (1980) Software for a cascade/parallel formant synthesizer. J Acoust Soc Am 67:971-995.

Klug A, Bauer EE, Hanson JT, Hurley L, Meitzen J, Pollak GD (2002) Response selectivity for species-specific calls in the inferior colliculus of mexican free-tailed bats is generated by inhibition. J Neurophysiol 88:1941-1954.

Koch U, Grothe B (1998) GABAergic and glycinergic inhibition sharpens tuning for frequency modulations in the inferior colliculus of the big brown bat. J Neurophysiol 80:71-82.

Konrad-Martin D, Dille MF, McMillan G, Griest S, McDermott D, Fausti SA, Austin DF (2012) Age-related changes in the auditory brainstem response. J Am Acad Audiol 23:18-35.

Kraus N, Chandrasekaran B (2010) Music training for the development of auditory skills. Nat Rev Neurosci 11:599-605.

Kraus N, Bradlow MA, Cunningham CJ, King CD, Koch DB, Nicol TG, McGee TJ, Stein LK, Wright BA (2000) Consequences of neural asynchrony: a case of auditory neuropathy. J Assoc Res Otolaryngol 1:33-45.

Krizman J, Marian V, Shook A, Skoe E, Kraus N (2012) Subcortical encoding of sound is enhanced in bilinguals and relates to executive function advantages. Proc Natl Acad Sci U S A 109:7877-7881.

Kuo BC, Yeh YY, Chen AJ, D’Esposito M (2011) Functional connectivity during top-down modulation of visual short-term memory representations. Neuropsychologia 49:1589-1596.

Liederman J, Frye R, Fisher JM, Greenwood K, Alexander R (2005) A temporally dynamic context effect that disrupts voice onset time discrimination of rapidly successive stimuli. Psychon Bull Rev 12:380-386.

Lister JJ, Roberts RA, Lister FL (2011) An adaptive clinical test of temporal resolution: age effects. Int J Audiol 50:367-374.

Luo H, Poeppel D (2007) Phase patterns of neuronal responses reliably discriminate speech in human auditory cortex. Neuron 54:1001-1010.

Maurizi M, Altissimi G, Ottaviani F, Paludetti G, Bambini M (1982) Auditory brainstem responses (ABR) in the aged. Scand Audiol 11:213-221.

Miller GA, Nicely PE (1955) An analysis of perceptual confusions among some English consonants. J Acoust Soc Am 27:338-352.

Nahum M, Nelken I, Ahissar M (2008) Low-level information and highlevel perception: the case of speech in noise. PLoS Biol 6:e126.

Nasreddine ZS, Phillips NA, Bédirian V, Charbonneau S, Whitehead V, Collin I, Cummings JL, Chertkow H (2005) The Montreal Cognitive Assessment, MoCA: a brief screening tool for mild cognitive impairment. J Am Gerontol Soc 53:695-699.

Parbery-Clark A, Skoe E, Kraus N (2009) Musical experience limits the degradative effects of background noise on the neural processing of sound. J Neurosci 29:14100-14107.

Parbery-Clark A, Strait DL, Anderson S, Hittner E, Kraus N (2011) Musical experience and the aging auditory system: implications for cognitive abilities and hearing speech in noise. PloS One 6:e18082.

Parbery-Clark A, Anderson S, Hittner E, Kraus N (2012) Musical experience offsets age-related delays in neural timing. Neurobiol Aging 33:1483.e1-e4.

Parthasarathy A, Cunningham PA, Bartlett EL (2010) Age-related differences in auditory processing as assessed by amplitude-modulation following responses in quiet and in noise. Front Aging Neurosci 2:152.

Pichora-Fuller MK, Schneider BA (1992) The effect of interaural delay of the masker on masking-level differences in young and old adults. J Acoust Soc Am 91:2129-2135.

Pichora-Fuller MK, Schneider BA, Macdonald E, Pass HE, Brown S (2007) Temporal jitter disrupts speech intelligibility: a simulation of auditory aging. Hear Res 223:114-121.

Prieto JJ, Peterson BA, Winer JA (1994) Morphology and spatial distribution of GABAergic neurons in cat primary auditory cortex (AI). J Comp Neurol 344:349-382.

Recanzone GH, Engle JR, Juarez-Salinas DL (2011) Spatial and temporal processing of single auditory cortical neurons and populations of neurons in the macaque monkey. Hear Res 271:115-122.

Rossi-Katz J, Arehart KH (2009) Message and talker identification in older adults: effects of task, distinctiveness of the talkers' voices, and meaningfulness of the competing message. J Speech Lang Hear Res 52:435-453.

Ruggles D, Bharadwaj H, Shinn-Cunningham BG (2011) Normal hearing is not enough to guarantee robust encoding of suprathreshold features im- 
portant in everyday communication. Proc Natl Acad Sci USA 108:15516-15521.

Ruggles D, Bharadwaj H, Shinn-Cunningham BG (2012) Why middle-aged listeners have trouble hearing in everyday settings. Curr Biol 22:1417-1422.

Russo FA, Ives DT, Goy H, Pichora-Fuller MK, Patterson RD (2012) Agerelated difference in melodic pitch perception is probably mediated by temporal processing: Empirical and computational evidence. Ear Hear 33:177-186.

Schatteman TA, Hughes LF, Caspary DM (2008) Aged-related loss of temporal processing: altered responses to amplitude modulated tones in rat dorsal cochlear nucleus. Neuroscience 154:329-337.

Schneider B, Pichora-Fuller MK (2001) Age-related changes in temporal processing: implications for speech perception. Semin Hear 22:227-240.

Schneider BA, Hamstra SJ (1999) Gap detection thresholds as a function of tonal duration for older and younger adults. J Acoust Soc Am 106:371-380.

Shamma SA (2011) Hearing impairments hidden in normal listeners. Proc Natl Acad Sci U S A 108:16139-16140.

Shinn-Cunningham BG, Best V (2008) Selective attention in normal and impaired hearing. Trends Amplif 12:283-299.

Skoe E, Kraus N (2010) Auditory brain stem response to complex sounds: a tutorial. Ear Hear 31:302-324.

Spivak LG, Malinoff R (1990) Spectral differences in the ABRs of old and young subjects. Ear Hear 11:351-358.

Strouse A, Ashmead DH, Ohde RN, Grantham DW (1998) Temporal processing in the aging auditory system. J Acoust Soc Am 104:2385-2399.

Tallon-Baudry C, Bertrand O, Delpuech C, Pernier J (1996) Stimulus specificity of phase-locked and non-phase-locked $40 \mathrm{~Hz}$ visual responses in humans. J Neurosci 16:4240-4249.

Tremblay KL, Piskosz M, Souza P (2002) Aging alters the neural representation of speech cues. Neuroreport 13:1865-1870.

Turner JG, Hughes LF, Caspary DM (2005) Affects of aging on receptive fields in rat primary auditory cortex layer V neurons. J Neurophysiol 94:2738-2747.

Vander Werff KR, Burns KS (2011) Brain stem responses to speech in younger and older adults. Ear Hear 32:168-180.

Vongpaisal T, Pichora-Fuller MK (2007) Effect of age on F0 difference limen and concurrent vowel identification. J Speech Lang Hear Res 50:1139-1156.

Walton JP, Frisina RD, O’Neill WE (1998) Age-related alteration in processing of temporal sound features in the auditory midbrain of the CBA mouse. J Neurosci 18:2764-2776.

Wang H, Turner JG, Ling L, Parrish JL, Hughes LF, Caspary DM (2009) Age-related changes in glycine receptor subunit composition and binding in dorsal cochlear nucleus. Neuroscience 160:227-239.

Wang M, Wu X, Li L, Schneider BA (2011) The effects of age and interaural delay on detecting a change in interaural correlation: the role of temporal jitter. Hear Res 275:139-149.

Willott JF, Parham K, Hunter KP (1988) Response properties of inferior colliculus neurons in middle-aged C57BL/6J mice with presbycusis. Hear Res 37:15-27.

Wingfield A, Tun PA, Koh CK, Rosen MJ (1999) Regaining lost time: adult aging and the effect of time restoration on recall of time-compressed speech. Psychol Aging 14:380-389.

Wong PC, Jin JX, Gunasekera GM, Abel R, Lee ER, Dhar S (2009) Aging and cortical mechanisms of speech perception in noise. Neuropsychologia 47:693-703

Yang Y, Liang Z, Li G, Wang Y, Zhou Y (2009) Aging affects response variability of V1 and MT neurons in rhesus monkeys. Brain Res 1274:21-27.

Yueh B, Shapiro N, MacLean CH, Shekelle PG (2003) Screening and management of adult hearing loss in primary care: scientific review. JAMA 289:1976-1985.

Zendel BR, Alain C (2012) Musicians experience less age-related decline in central auditory processing. Psychol Aging 27:410-417.

Zhu J, Garcia E (1999) The Wechsler Abbreviated Scale of Intelligence (WASI). New York: Psychological Corporation. 